\title{
Complex Langevin simulation for QCD-like models
}

\author{
Gert Aarts, Lorenzo Bongiovanni, Erhard Seiler, Dénes Sexty, lon-Olimpiu \\ Stamatescu*t \\ FEST \\ E-mail: stamates@thphys.uni-heidelberg.de
}

\begin{abstract}
We first test the Complex Langevin method (CLE) on various simple models. We then introduce the method of Gauge Cooling to control the dynamics of the process and ensure thin distributions in the imaginary direction. We finally apply CLE with gauge cooling to a QCD-related lattice model (HQCD) and compare the results by CLE and by a refined Reweighting method (rRW). Very good agreement is found in all regions of physical interest.
\end{abstract}

31 st International Symposium on Lattice Field Theory - LATTICE 2013

July 29 - August 3, 2013

Mainz, Germany

\footnotetext{
${ }^{*}$ Speaker.

${ }^{\dagger}$ Partial support by the Deutsche Forschungsgemeinschaft is thankfully acknowledged
} 


\section{Motivation and program}

The Complex Langevin Equation (CLE) has the potential to simulate lattice models for which usual importance sampling fails. In many cases, especially for QCD at non-zero density, the CLE in principle provides the (only) model independent procedure.

The real Langevin Equation (LE) is a well studied stochastic process. Its redefinition as CLE is more involved. To develop it to a reliable method is both rewarding and tough. Our program is:

- Define and study the properties of the CLE, test CLE for simple models.

- Apply CLE to realistic models aiming at full QCD at non-zero chemical potential [1].

\section{The Langevin equation for real models}

The LE for a real field $\varphi(x)$ evolving in the process time $t$ ("Langevin" time, here discretized) is:

$$
\begin{aligned}
& \delta \varphi(x ; t)=K[\varphi(x ; t)] \delta t+\eta(x ; t) \\
& \langle\eta(x, t)\rangle=0, \quad\left\langle\eta(x, t) \eta\left(x_{1}, t_{1}\right)\right\rangle=2 \delta t \delta_{x, x_{1}} \delta_{t, t_{1}}
\end{aligned}
$$

( $\delta t$ : time step, Ito calculus) with the associated Fokker-Planck equation (FPE)

$$
\partial_{t} P(\varphi, t)=\partial_{\varphi}\left(\partial_{\varphi}-K\right) P(\varphi, t) .
$$

If the drift $K=-\partial_{\varphi} S$ with $S$ a positive definite action we then have asymptotically

$$
t \rightarrow \infty \quad P(\varphi, t) \rightarrow P_{a s}(\varphi)=\frac{1}{Z} \exp (-S), \quad Z=\int[d \varphi] \exp (-S) .
$$

For positive measure the LE is well defined and comparable with Monte Carlo. In the presence of a sign problem LE may have difficulties. One can study this in simple models and devise systematic cures [2] overcoming old "disasters" [3]. The problems, however, may be inherited in the CLE.

\section{Set up for the CLE}

For a complex action the drift is also complex and this automatically provides an imaginary part for the field. This implies setting up the problem in the complexification of the original manifold $R^{n} \longrightarrow C^{n}$ or $S U(n) \longrightarrow S L(n, C)$. The CLE then amounts to two related, real LE with independent noise terms - here for just one variable $x \rightarrow z=x+i y$ and with $K=-\partial_{z} S(z)$ :

$$
\begin{aligned}
\delta z(t)= & K(z) \delta t+\sqrt{N_{R}} \eta_{R}+\mathrm{i} \sqrt{N_{I}} \eta_{I} \\
\text { i.e. } & \delta x(t)=\operatorname{Re} K(z) \delta t+\sqrt{N_{R}} \eta_{R}(t), \quad \delta y(t)=\operatorname{Im} K(z) \delta t+\sqrt{N_{I}} \eta_{I}(t) \\
\left\langle\eta_{R}\right\rangle= & \left\langle\eta_{I}\right\rangle=0,\left\langle\eta_{R} \eta_{I}\right\rangle=0, \quad\left\langle\eta_{R}^{2}\right\rangle=\left\langle\eta_{I}^{2}\right\rangle=2 \delta t, \quad N_{R}-N_{I}=1
\end{aligned}
$$

The probability distribution $P(x, y ; t)$ realized in the process evolves according to a real FPE:

$$
\partial_{t} P(x, y, t)=L^{T} P(x, y, t), \quad L=\left(N_{R} \partial_{x}+\operatorname{Re} K(z)\right) \partial_{x}+\left(N_{I} \partial_{x}+\operatorname{Im} K(z)\right) \partial_{y}
$$

One can also define a complex distribution $\rho(x, t)$

$$
\partial_{t} \rho(x, t)=L_{0}^{T} \rho(x, t), \quad L_{0}=\left(\partial_{x}+K(x)\right) \partial_{x}
$$


with the asymptotic solution $\rho(x) \simeq \exp (-S(x))$ and formally prove for analytic observables $O(z)$

$$
\int O(x+i y) P(x, y ; t) d x d y=\int O(x) \rho(x ; t) d x .
$$

The formal proof has, however, loopholes related among others to a too wide $P(x, y, t)$ in $y$ [4]. This width may be enhanced by an imaginary part in the noise, therefore one usually uses $N_{I}=0$.

\section{Problems and models}

Very many studies for CLE have appeared since the original papers of Parisi and of Klauder [5] including critical analysis, cf e.g. [3]. The problems studied in our group include: Real time simulations, Chemical potential, $\theta$-term. We here address QCD with chemical potential:

$$
\begin{aligned}
Z & =\int D U \operatorname{det} W \mathrm{e}^{-S_{Y M}}, \\
W & =1-\kappa \sum_{i=1}^{3}\left(\Gamma_{+i} U_{x, i} T_{i}+\Gamma_{-i} U_{x, i}^{-1} T_{-i}\right)-\kappa \gamma\left(\mathrm{e}^{\mu} \Gamma_{+4} U_{x, 4} T_{4}+\mathrm{e}^{-\mu} \Gamma_{-4} U_{x, 4}^{-1} T_{-4}\right)
\end{aligned}
$$

Wilson fermions, $T$ : lattice translations, $\Gamma_{ \pm \mu}=1 \pm \gamma_{\mu}, \kappa=1 /(2 M+8), M$ bare mass, $\gamma$ bare anisotropy. The temperature is introduced as $a T=\frac{\gamma}{N_{\tau}}$. We have $\operatorname{det} W(\mu)=[\operatorname{det} W(-\mu)]^{*}$. CLE does not have an overlap problem such as the reweighting methods (RW) and does not involve approximations like expansion methods: The ensemble is generated at the actual values of the parameters without restriction in the latter. The problems one encounters with CLE can be:

1) Accumulation of numerical errors. Typical effect: run-aways, divergence of some quantities. This can be efficiently solved by adaptive step size (which we use throughout our analysis) [6].

2) insufficient fall off of $P(x, y)$ in the non-compact directions can lead to imprecise sampling and also can spoil the formal proof of equivalence. This can be to a good extent approached by controlling the dynamics of the process, e.g. by gauge cooling for gauge models (see below).

3) Non-holomorphy of the drift can invalidate the formal proof of equivalence, e.g. poles of $K(z)$ coming from zeroes of the measure, $\rho(z)$. To this challenge we only have partial answers [2].

To control the reliability we implement a number of checks (Consistency Conditions CC [7] combinations of observables which should vanish identically in the correct case -, monitoring the distributions) and stabilizing procedures generalizing the CLE [8].

\section{One link effective model}

A paradigmatic effective model is an $\mathrm{SU}(3)$ model with one link $U$. Diagonalizing $U$ we obtain a reduced model with the reduced Haar measure in the three diagonal exponents $w_{i}$

$$
\begin{aligned}
Z= & \int[d w] \rho(w), \quad \rho(w)=\mathrm{e}^{-S_{Y M}} H(w) D(w) \tilde{D}(w), \quad w_{1}+w_{2}+w_{3}=0 \\
& S_{Y M}=-\frac{\beta}{2} \sum_{i=1}^{3}\left(\alpha_{i} e^{i w_{i}}+\frac{1}{\alpha_{i}} e^{-i w_{i}}\right), \quad H=\sin ^{2} \frac{w_{2}-w_{3}}{2} \sin ^{2} \frac{w_{3}-w_{1}}{2} \sin ^{2} \frac{w_{1}-w_{2}}{2}, \\
& D=1+C \operatorname{tr} U+C^{2} \operatorname{tr} U^{-1}+C^{3}, C=2 \kappa \mathrm{e}^{\mu} ; \quad \tilde{D}=1+\tilde{C} \operatorname{tr} U^{-1}+\tilde{C}^{2} \operatorname{tr} U+\tilde{C}^{3}, \tilde{C}=2 \kappa \mathrm{e}^{-\mu} \\
& K_{i}(w)=K_{Y M, i}+H^{-1} \partial_{w_{i}} H+D^{-1} \partial_{w_{i}} D+\tilde{D}^{-1} \partial_{w_{i}} \tilde{D} .
\end{aligned}
$$


The $\alpha$ 's simulate the "staples" of the neighbours. As a general remark, observe that one first needs to complexify the variables, here in going from su(3) to sl(3,C), before deriving the drift $K(w)$. $K(w)$ is generally meromorphic due to possible poles from the zero's of the determinants.

We find that correct results are obtained if the flow does not drift too far in the non-compact directions - Fig. 1. This effect must be monitored and suggests possibilities to redesign the process to control the skirt of the distribution. For $\alpha$ complex, far from 1, CLE departs from the exact results (left plot, solid lines). This correlates here with wide skirts of the $\operatorname{Im} w$-distributions (right plot). Another source of non-reliability are the poles. This is seen in this model [2] as well as in more complex models where the determinants may have zeroes [9].
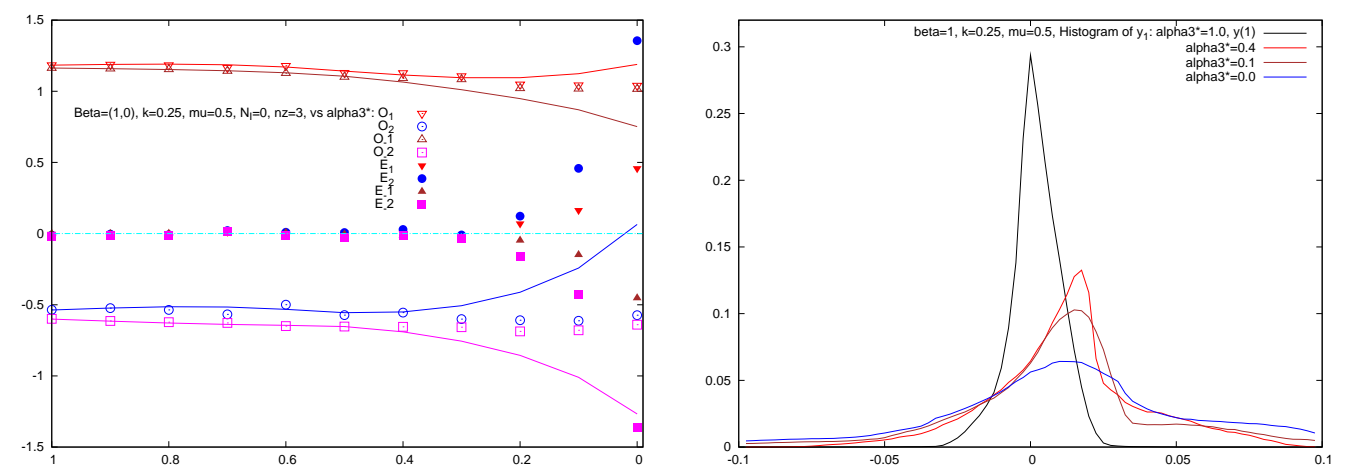

Figure 1: Effective model: Observables $O_{q}=e^{i q z}, \operatorname{CC}$ 's $E_{q}, q= \pm 1, \pm 2$ vs $\operatorname{Re} \alpha_{i}$ and the $y$-distribution.

\section{Many Links models and Gauge Cooling.}

To see the effect of many variables we consider an exactly soluble Polyakov chain model:

$$
-S=\left(\beta+2 \kappa \mathrm{e}^{\mu}\right) P+\left(\beta+2 \kappa \mathrm{e}^{-\mu}\right)^{*} P^{-1}
$$

with $\mathrm{P}=\operatorname{Tr}\left(U_{1} \cdots U_{N}\right), N$ up to 1024 . The process runs in all $8 N$ (complex) "angles" $A_{i}^{a}$ :

$$
\delta A_{i}^{a}=\varepsilon K_{i}^{a}(U)+\sqrt{\varepsilon} \eta, U_{i} \rightarrow \mathrm{e}^{i \sum_{a} \lambda_{a} \delta A_{i}^{a}} U_{i}
$$

with holomorphic drift $K$. For large $N$ we observe, however, wrong evolution even in the real case $(\mu=0)$ if we set up the process as CLE, although the drift and noise are real! We quantify this by measuring the departure of the links from unitarity with a unitarity norm, e.g.

$$
\mathscr{U}=\sum_{\text {links }}\left[\frac{1}{2} \operatorname{Tr}\left(U U^{\dagger}+U^{-1} U^{-1 \dagger}\right)-3\right] .
$$

This effect - Fig. 2, left plot - suggests that numerical imprecisons may trigger unstable modes leading away from the real axis. For simpler models fixing the gauge was observed to help [10]. Using the gauge symmetry of the problem we now define a general Gauge Cooling procedure to bring the system as near as possible to the unitary manifold. This proceeds by successive noncompact gauge transformations along the gradient of the unitarity norm $\mathscr{U}$ on the gauge orbits

$$
R_{k}=e^{-\alpha \varepsilon d \mathscr{U}}, \quad U_{k} \rightarrow R_{k} U_{k}, \quad U_{k-1} \rightarrow U_{k-1} R_{k}^{-1}
$$



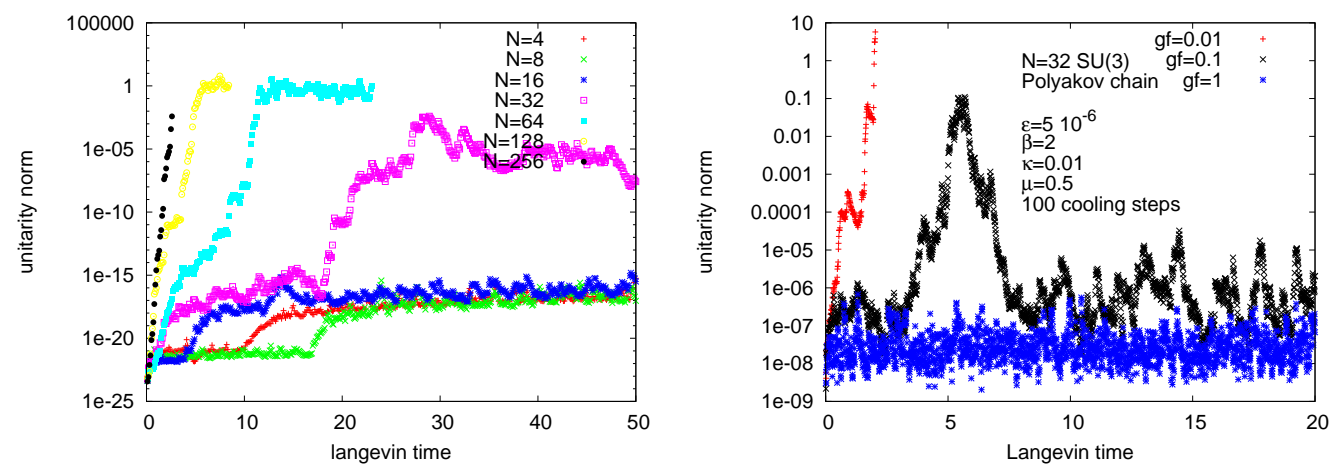

Figure 2: Polyakov chain model, evolution of the unitarity norm in Langevin time. Left plot: $\mu=0$, various chain lenths, no cooling. Right plot: $\mu>0, N=32$, various coolings.

with $\alpha$ : the strength of the gauge force, $\varepsilon$ : Langevin step size. For $\mu>0 \mathscr{U}$ should not be 0 but stabilize. This we see after gauge cooling (large $\alpha$ and/or many cooling steps) - Fig. 2, right plot. Then also the results are correct and the non-compact distributions narrow - Fig. 3.
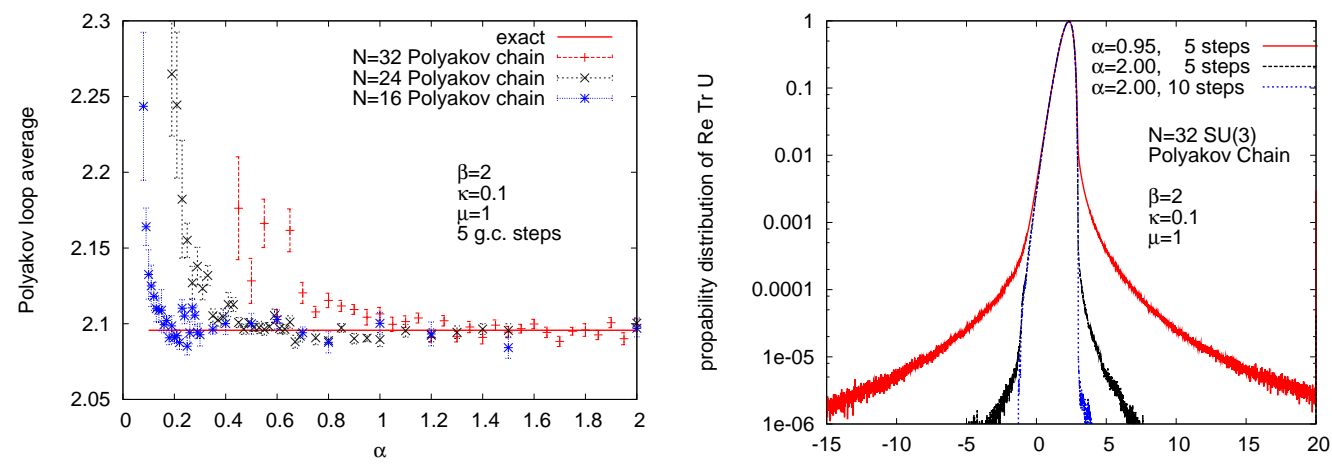

Figure 3: Polyakov chain model. Left plot: Polyakov loop average for various chain lengths as function of the cooling strength. Right plot: Polyakov loop distribution for different amount of cooling.

\section{Heavy QCD at non-zero chemical potential.}

Taking in the hopping parameter expansion of the fermionic determinant the double limit [11]

$$
\kappa \rightarrow 0, \mu \rightarrow \infty, \zeta=\kappa \mathrm{e}^{\mu}: \text { fixed }
$$

we obtain an approximation for QCD at large mass and chemical potential. In this limit only the Polyakov loops survive and the determinant factorizes. This can be used, e.g. in refined reweighting (rRW) simulations (cf [12], where also the relevant formulae and the next corrections are given). Using also the inverse Polyakov loops (which in the above limit are not present) one obtains a model by itself, HQCD, which can be followed also away from this limit [13]. Both a CLE and an accordingly "symmetrized" rRW approach can be implemented for this model. 
Using CLE we observe for HQCD the same effects as for the Polyakov chain. The following results are obtained with gauge cooling, which ensures a stabilized unitarity norm [14]. We measure plaquettes, Polyakov loops $\mathrm{P}$ and $\mathrm{P}^{-1}$, baryon density $n$ and the average phase:

$$
\langle\exp (2 i \phi)\rangle \equiv\left\langle\operatorname{det} \mathbf{M}(\mu) \operatorname{det} \mathbf{M}(-\mu)^{-1}\right\rangle .
$$

The full YM action is used. We observe stable results for all $\mu$ all the way from $\mu=0$ up to deeply in the saturation regime - Fig. 4. The results show the expected behaviour of the Polyakov loops and baryon density and that the method work very well also in the region where the phase factor is practically 0 (see also [15] for resummed strong coupling results).
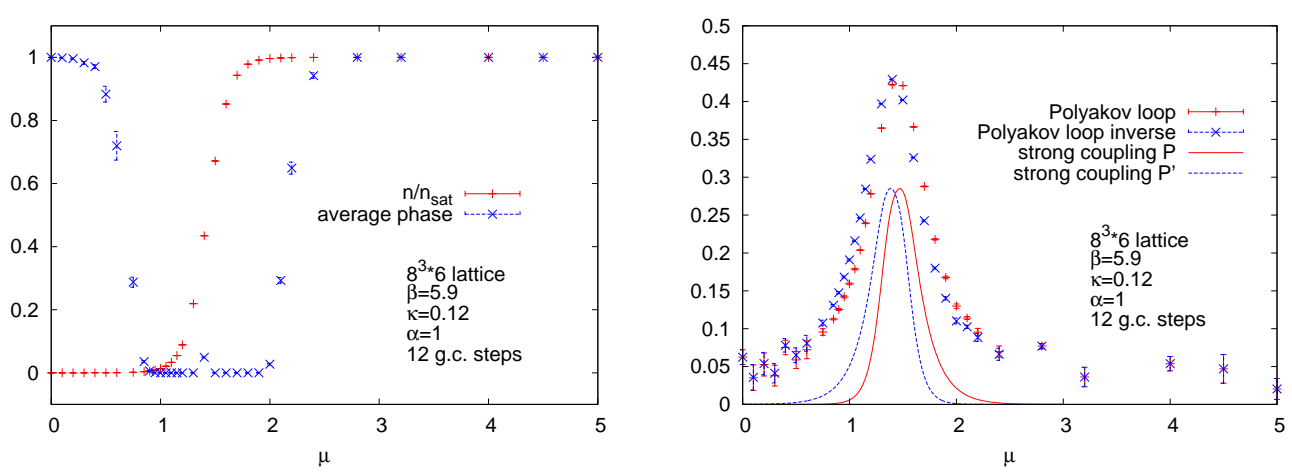

Figure 4: HQCD model, CLE with gauge cooling: Baryon density and average phase (left plot) and Polyakov and inverse Polyakov loop averages (right plot) vs $\mu, 8^{3} 6$ lattice.

In the following we compare the CLE results with those from the symmetrized version of rRW - Fig. 5. Both plaquettes and Polyakov loops agree extremely well for all values of $\mu$ in the deconfined region $\left(\beta=5.9,6^{4}\right.$ lattice - the large errors affect rRW at large $\mu$ ). At fixed $\mu=0.85,6^{4}$ the agreement persists except for $\beta<5.7$, indicating possible difficulties of the CLE. This effect seems, however, to be $\beta$ and not scale dependent, for large lattices we can reach deeply into the confining region (compare the $10^{4}$ lattice, where the transition is expected at $\beta \simeq 5.9$ ) - Fig. 6 . The excellent agreement between these two completely different methods is a strong argument for the validity of both of them in most regions of physial interest. For a general review see [16].

\section{References}

[1] D. Sexty, arXiv:1307.7748 (2013)

[2] G. Aarts, L. Bongiovanni, E. Seiler, D. Sexty, I.-O. Stamatescu, work in progress

[3] J. Ambjorn, M.Flensburg, C. Peterson, Nucl. Phys. B 275 (1986) 375

[4] G. Aarts, E. Seiler, I.-O. Stamatescu, Phys. Rev. D 81 (2010) 054508 [arXiv 0912.3360]

[5] G. Parisi, Phys. Lett. 131 B (1983 393; J.R. Klauder, Acta Phys. Austriaca, 25 (1983) 251

[6] J. Flower, S.W. Otto, S. Callahan, Phys. Rev. D 34 (1986) 598; G. Aarts, F.A. James, E. Seiler, I.-O. Stamatescu, Phys. Lett. B 687 (2010) 154 [arXiv:0912.0617] 

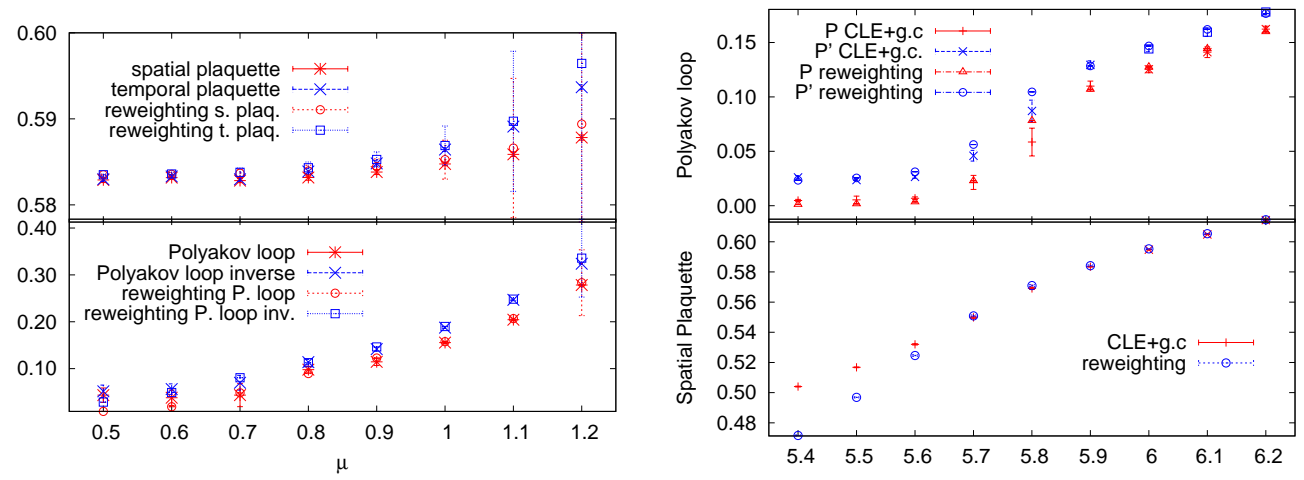

Figure 5: HQCD model: CLE with gauge cooling, plaquette and Polyakov loop averages vs $\mu$ at $\beta=5.9$ (left plot) and vs $\beta$ at $\mu=0.85$ (right plot), $6^{4}$ lattice, compared with rRW results.
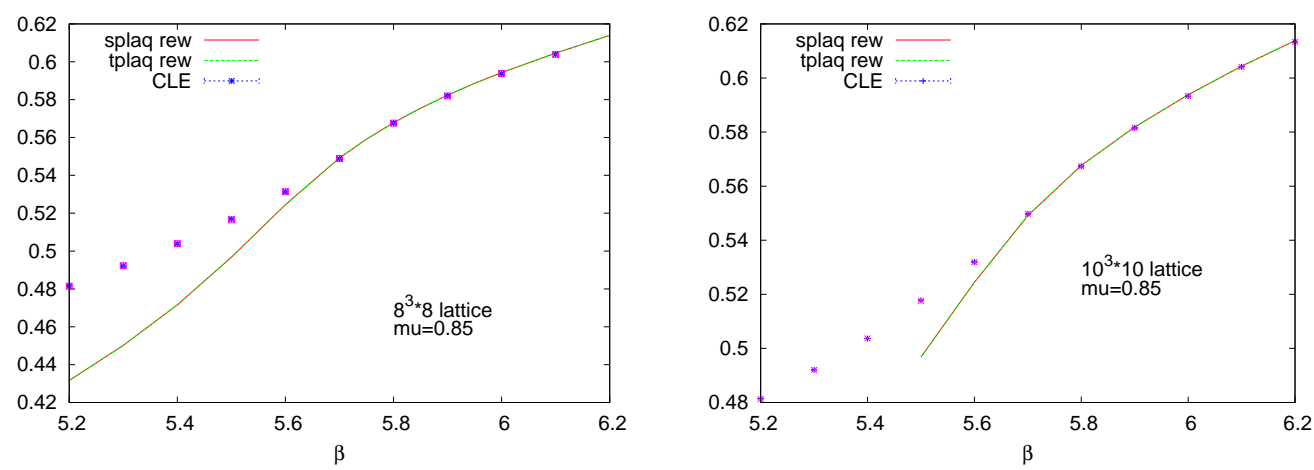

Figure 6: HQCD model: CLE with gauge cooling, plaquette averages at $\mu=0.85$ compared with the rRW results for two lattice sizes: $8^{4}$ (left) and $10^{4}$ (right), compare also with previous figure.

[7] G. Aarts, F.A. James, E. Seiler, D. Sexty, I.-O. Stamatescu, Eur.Phys.J. C 71 (2011) 1756 [arXiv:1101.3270]

[8] G. Aarts, F.A. James, E. Seiler, J.M. Pawlowski, D. Sexty, I.-O. Stamatescu, JHEP 1303 (2012) 073 [arXiv:1212:5231]

[9] A. Mollgaard and K. Splittorff, arXiv:1309.4335 (2013)

[10] J. Berges and D. Sexty, Nucl. Phys. B 799 (2008) 306 [arXiv:0708.0779]

[11] I. Bender, T. Hashimoto, F. Karsch, V. Linke, A. Nakamura, M. Plewnia, I.-O. Stamatescu, W. Wetzel, Nucl. Phys. Proc. Suppl. 26 (1992) 323

[12] R. De Pietri, A. Feo, E. Seiler, I.-O. Stamatescu, Phys. Rev. D 76 (2007) 114501 [arXiv:0705.3420]

[13] G. Aarts and I.-O. Stamatescu, JHEP 0809 (2008) 018 [arXiv:0807.1597]

[14] E.Seiler, D. Sexty and I.-O. Stamatescu, Phys. Lett. B 723 (2013) 213 [arxiv: 1211.3709]

[15] M. Fromm, J. Langelage, S. Lottini, and O. Philipsen, JHEP 1201 (2012) 042 [arXiv:1111.4953].

[16] G. Aarts, L. Bongiovanni, E. Seiler, D. Sexty, I.-O. Stamatescu, Eur.Phys.J. A 49 (2013) 89, [arXiv:1303.6425] 